\title{
Comparison of remdesivir and favipiravir - the anti-Covid-19 agents mimicking purine RNA constituents
}

\author{
Faiha M. Al-Ardhi', Ladislav Novotny ${ }^{\mathrm{a}}$, Adel Alhunayann ${ }^{\mathrm{b}}$, Naser F. Al-Tannak ${ }^{\mathrm{a}}$
}

By December 2019, humanity was challenged by a new infectious respiratory disease named coronavirus disease of 2019 or COVID-19. This is a viral infection based on the presence of the previously non-problematic coronavirus with assigned number 2 . This virus causes severe acute respiratory distress and is known now as SARS-CoV2. Since SARSCoV2 is an RNA virus, remdesivir and favipiravir, both broad-spectrum RNA polymerase inhibitors, were repurposed for treating COVID-19 patients. Remdesivir and favipiravir are antimetabolites, and they are structurally related to the naturally occurring structural elements of RNA. Both agents are prodrugs and must be activated intracellularly to exert their effects through numerous and different mechanisms of action. Efforts have been exerted to determine their efficacy and safety against COVID-19 through clinical trials. Clinical trials have shown an association of remdesivir with increased frequency of adverse effects (in comparison to favipiravir). Nevertheless, the data obtained with remdesivir resulted in its approval by the FDA on the $22^{\text {nd }}$ of October 2020 for COVID-19 treatment. At present, remdesivir is being recommended by several treatment guidelines for the treatment of COVID-19 patients. The evidence in favor of favipiravir is compromised by the small number and low-quality of trials conducted. Favipiravir has shown various benefits when administered in mild and moderate cases of COVID-19, while remdesivir was more beneficial in more severe cases of the disease. Since the two agents are suitable for different groups of patients, both drugs can play a significant role in fighting this pandemic. The goal of this work is to summarize the information available on two antimetabolites - remdesivir and favipiravir - and to compare clinical experience obtained so far with these two agents in COVID-19 patients.

Key words: remdesivir, favipiravir, Covid-19, antimetabolites, RNA-polymerase inhibitors, respiratory syndrome

Received: July 16, 2021; Revised: October 26, 2021; Accepted: November 2, 2021; Available online: November 15, 2021 https://doi.org/10.5507/bp.2021.063

(c) 2022 The Authors; https://creativecommons.org/licenses/by/4.0/

${ }^{a}$ Department of Pharmaceutical Chemistry, Faculty of Pharmacy, Health Science Center, Kuwait University, P.O. Box 24923, Safat 13110, Kuwait

${ }^{b}$ Faculty of Medicine, Health Science Center, Kuwait University, P.O. Box 24923, Safat 13110, Kuwait

All authors contributed equally to this work

Corresponding author: Ladislav Novotny, e-mail: ladislav.novotny@ku.edu.kw

\section{INTRODUCTION}

In 2019, a new health threat had emerged in China. The disease caused by a coronavirus infection is currently recognized as COVID-19 infection. It was first identified as pneumonia cases. The number of infection cases increased enormously causing a pandemic declared as such at the beginning of the next year ${ }^{1}$. COVID-19 is a new type of infection of the respiratory tract inflicted on people by SARS-CoV2 infection. At first, knowledge about the virus and the potential treatment was guided by the previous experience with two respiratory diseases, namely severe acute respiratory syndrome (SARS) and the Middle East respiratory syndrome (MERS). SARS appeared in 2002 and MESR in 2012. These three diseases are caused by beta-coronaviruses of the Coronaviridae family $^{2}$. Genetic analysis of the coronaviral agent supported the assumption of bats being the origin of the virus and the Malayan pangolin an intermediate host. Complex recombination events accompany the transmission between these three species. The viral genome is formed by a single-stranded positive-sense ribonucleic acid (gRNA) that serves as a messenger RNA (mRNA) for the synthesis of subgenomic RNA ( $\operatorname{sgRNA}$ ). Both gRNA and sgRNA are translated into structural proteins. These proteins are essential for virus functioning and they are named according to their position in the structure of the virus ( $\mathrm{S}$ for spike protein, $\mathrm{M}$ for membrane protein, and $\mathrm{E}$ for envelope protein), and also $\mathrm{N}$ for nucleocapsid proteins. Other proteins coded by viral RNA are nonstructural proteins and accessory proteins ${ }^{3}$. The assembly of these structural proteins produces a helical viral nucleocapsid covered by an oval-shaped envelope with glycoproteins spikes ${ }^{2}$. These spike proteins bind to the human angiotensin converting enzyme 2 (ACE2) receptor on the cell membrane for attachment and entry. The viron-ACE2 interaction also plays a role in COVID-19 pathogenesis through the imbalance of renin-angiotensin system ${ }^{4}$. SARS-CoV2 is mainly transmitted through droplets from infected people, both symptomatic and asymptomatic, or by contact with infected objects. Recent evidence suggests that fecal-oral and airborne transmis- 
sions are also possible ${ }^{5}$. The virus incubation period is 2 to 14 days. Infected-with-COVID-19 patients suffer from fatigue, cough, headache, and fever as main symptoms. Hemoptysis and diarrhea are observed to a lesser extent. In severe cases, the patients suffer from severe acute respiratory distress demonstrating itself through pneumonia, and shortness of breath because of severe alveolar damage and other histological changes of the lungs. These can eventually lead to death. Older patients or middle-aged patients with chronic diseases (i. e. diabetes or hypertension) are at an increased risk regarding the severity of the symptoms and failure of the respiratory system and their prognosis is, in general, worse compared to younger and healthier patients ${ }^{2,5}$. At the beginning of COVID-19 pandemic, no effective drugs or vaccines were available for this new infection. Because of this, several drugs active against other RNA viruses were tested for their activity against the new infection. Remdesivir represents such an antiviral substance. Other tested molecules were hydroxychloroquine, ivermectin, and lopinavir/ ritonavir, and some others. Later, corticosteroids and immunosuppressants were used as exaggerated immunity plays a major role in the pathology of COVID-19. Other therapeutic options are monoclonal antibodies against SARS-CoV-2 (i. e. bamlanivimab or casirivimab plus imdevimab) (ref. ${ }^{6}$ ). Recombinant ACE2- immunoglobulin was also investigated as potential agents against SARS-CoV2 (ref. ${ }^{4}$ ). Clinical data about the effectiveness and safety of therapeutic alternatives for COVID-19 are continuously updated ${ }^{6}$. This review aims to explore and compare the properties and information on two antimetabolites - a nucleotide analog remdesivir and a nucleobase analog favipiravir - because of the benefits that they may provide as a part of COVID-19 treatment.

\section{THE CONCEPT OF ANTIMETABOLITES AND THEIR USES IN ANTIVIRAL THERAPY}

Many antimetabolites have been approved for clinical use in the last fifty years. They are widely utilized in anticancer and antivirals therapies. An antimetabolite is a synthetic molecule structurally related to the naturally occurring substrate(s). The most active antimetabolites take part in a cellular metabolic pathway by mimicking one of the nucleobases or their corresponding nucleoside or nucleotide. Antimetabolites deplete or inhibit essential cellular enzymes (i.e. kinases, polymerases, or ribonucleotide reductase), both human and viral. Additionally, they can incorporate directly into the newly synthesized strands of nucleic acids. This leads to the obligate or delayed termination of chain elongation, the induction of apoptosis, or the accumulation of mutants ${ }^{7,8}$. These events occur because any structural modification of the natural nucleosides/nucleotides can have a major and significant effect on the way they interact with the binding sites of the named enzymes. The synthetic nucleoside/nucleotide analogs may differ in bioavailability, delivery to the active sites, selectivity, and safety. The drug industry aims at the identification of modifications improving the molecule effect or stability. Modifications may be based on modifying the base, sugar, or phosphate group by adding, removing, replacing, or repositioning an atom or functional group ${ }^{7.9}$. Currently, antimetabolites are essential in the therapy of viral infections caused by various viruses (hepatitis B and $\mathrm{C}$ and HIV). Nucleoside/ nucleotide analogs act on many viruses. However, in these two cases (remdesivir and favipiravir), resistance does not develop since the structure of their binding sites to SARS-CoV-2 is very conserved ${ }^{10}$. Therapeutically useful antiviral antimetabolites should possess high selectivity for viral enzymes over mammalian

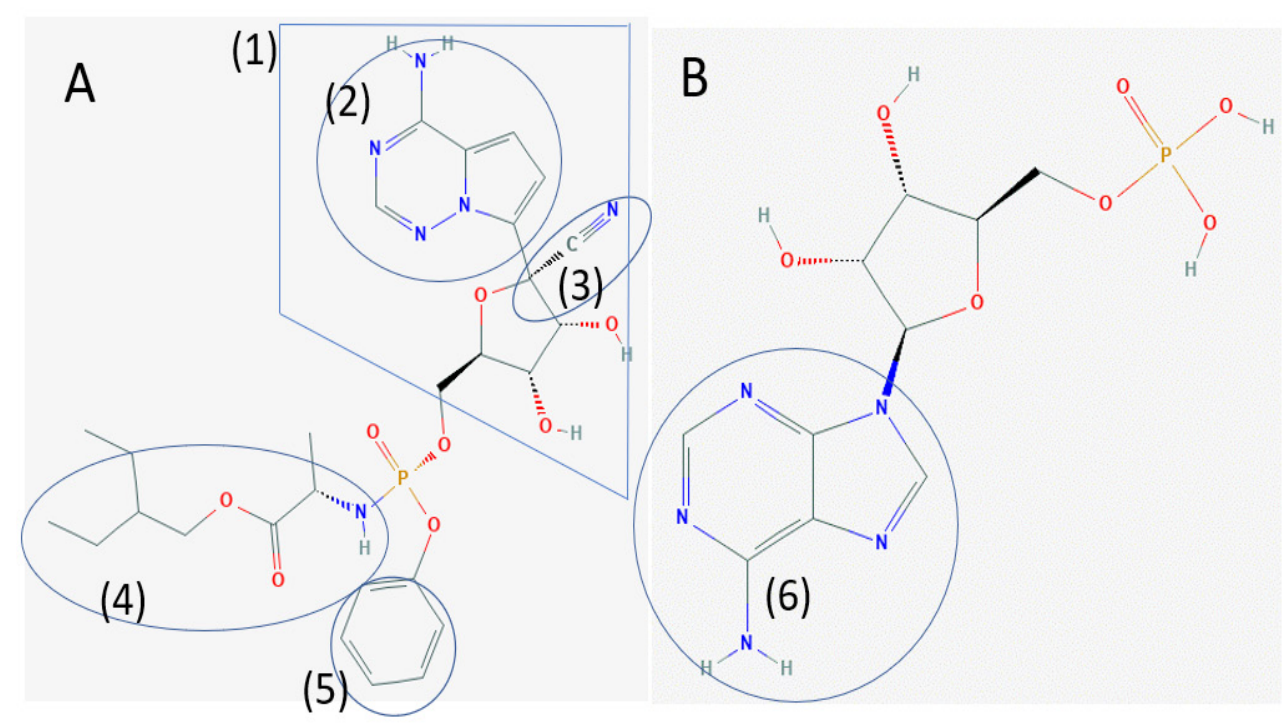

Fig. 1. Chemical structure of remdesivir ${ }^{41}$ (A) and adenosine 5'-monophosphate (B).

A: (1) Modified C-adenosine (GS-441524), (2) modified adenine nucleobase, (3) cyano group at C-1 of the ribose, (4) amino acid ester substituent of the phosphoric acid moiety, (5) phenyl substituent of the phosphoric acid moiety. B: (6) adenine 
enzymes ${ }^{7}$. Antiviral antimetabolites inhibit viral replication by targeting polymerase or reverse transcriptase enzymes. Polymerase inhibitors are further subdivided into DNA polymerase inhibitors (i.e., acyclovir), and RNA polymerase inhibitors (i.e., remdesivir or favipiravir) (ref. $\left.{ }^{11}\right)$.

\section{Chemical properties of remdesivir and favipiravir}

Remdesivir and favipiravir are two structurally unrelated antimetabolites. Both agents mimic the structural elements of the viral genome and subsequently inhibit RNA polymerase. The action of these two antivirals is based on their chemical structure.

Remdesivir (GS-5734) is an adenosine monophosphate analog. It has a molecular formula of $\mathrm{C}_{27} \mathrm{H}_{35} \mathrm{~N}_{6} \mathrm{O}_{8} \mathrm{P}$. The IUPAC name of this compound is 2-ethylbutyl-(2S)2-[[[(2R,3S,4R,5R)-5-(4-aminopyrrolo[2,1-f][1,2,4] triazin-7-yl)-5-cyano-3,4-dihydroxyoxolan-2-yl]methoxyphenoxy-phosphoryl]amino]-propanoate. Fig. 1 shows that it is composed of a modified $\mathrm{C}$-adenosine nucleoside (1), which consists of a modified adenine nucleobase (2) and a substituted ribose sugar, and a phosphoramidite derivative. Within the GS-5734 structure, the manufacturer (Gilead Sciences) gave the modified C-adenosine nucleoside (1) the identifier GS-441524. Comparing the two structures (A and B), GS-441524 and adenosine have the same aldopentose sugar (ribofuranose), naturally present in RNA-nucleosides. However, they differ in the number and positions of nitrogen atoms within the nucleobases (2 and 6). These changes affect the hydrogen bonds and how the molecule interacts with its binding site. Moreover, the glycosidic O-C-N bond that links the nucleobase to the ribose sugar is modified to be an O-C-C bond, hence the name C-nucleoside. The new arrangement stabilizes the glycosidic bond and allows for 1'-cyano substitution. The cyano group substituted at $\mathrm{C}-1$ of the ribose sugar (3) is essential for remdesivir activity. Studies showed that the 1'-cyano binding site is only present in the viral RNA polymerase. This increases the drug selectivity toward viral polymerase over human polymerase. This substitution is also responsible for remdesivir broad activity against a wide range of viruses. GS-441524 can be referred to as 1'-cyano substituted 4-aza-7,9-dideazaadenosine C-nucleoside ${ }^{9,12}$. A phosphate group present in remdesivir is essential for and addition of the first phosphate group as the synthesis of monophosphate of a nucleoside is normally the rate-limiting step of nucleoside transformation to various nucleotides. This is true for nucleoside-based antimetabolites. Furthermore, substitutions of the phosphoric acid moiety of remdesivir with a phenyl (5) and an amino acid ester (ester of 2-ethylbutanol and 2-aminopropanoic acid - (4)) decrease or mask polarity of the phosphate and protect the drug from cellular enzymes, including phosphatases. The increased lipophilicity enables the compound to passively pass the cellar membrane while natural nucleotides enter the cell through specific nucleotides transporters. Subsequently, remdesivir is efficiently delivered into cells and is activated intracellularly ${ }^{7,12}$.

Favipiravir, or T-705, is a pyrazine analog with the

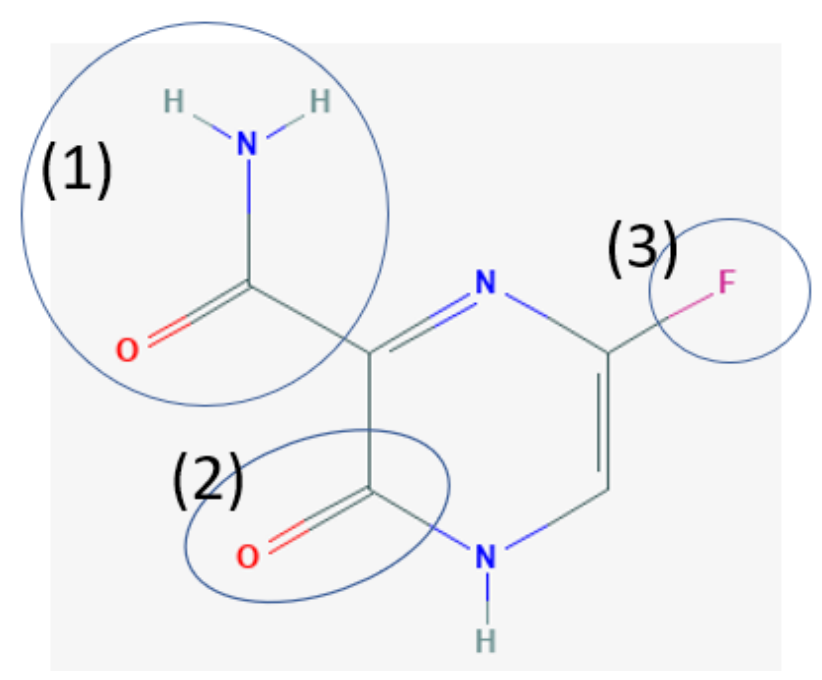

Fig. 2. Chemical structure of favipiravir ${ }^{42}$.

(1) Carboxamide moiety, (2) carbonyl group, (3) fluorine

molecular formula of $\mathrm{C}_{5} \mathrm{H}_{4} \mathrm{FN}_{3} \mathrm{O}_{2}$. Its name according to IUPAC is 5-fluoro-2-oxo-1H-pyrazine-3-carboxamide (Fig.2). The pyrazine ring in the structure of favipiravir possesses a carbonyl at C2 (2) leading to keto-enol tautomerism. The enol form is more predominant, stable, and biologically active as purine nucleobases mimetic ${ }^{13}$. However, the hydroxyl group present in the enol form is important for the recognition and enzymatic activation of favipiravir ${ }^{14}$. Another interesting feature of favipiravir is the presence of the carboxamide moiety in its structure at C-3 (1). This moiety can rotate, making favipiravir a pseudo-base of the two purine nucleobases - adenine and guanine $^{13}$. The positions of nitrogen atoms differ from the natural nucleobases it mimics. This affects how the molecules interact through hydrogen bonds ${ }^{9}$. Lastly, fluorine at C5 (3) replaces isosterically the hydrogen atom and, at the same moment, it functions as isopolar substitution of the hydroxyl due to its size and high electronegativity. Fluorine also increases the stability of favipiravir ${ }^{15}$.

\section{Mechanisms of action of remdesivir and favipiravir against Covid-19 \\ Bioactivation}

The increased lipophilicity enables remdesivir to pass the cellular phospholipid membrane. After that, remdesivir is activated. Initially, the molecule is hydrolyzed by esterases into carboxylate moiety that cyclizes into phosphonate while the phenoxide group is removed. The formed cyclic anhydride is unstable and re-open by hydrolysis into an alanine metabolite, GS-704277. Phosphoramidase-type enzymes hydrolyze the GS-704277 $\mathrm{N}-\mathrm{P}$ bond liberating the nucleoside monophosphate (MP) (i.e., nucleotide analog) that cannot diffuse out of the cell due to high polarity. Lastly, host cell nucleotide kinases further phosphorylate the molecule into the triphosphate responsible for the intracellular action of activated remdesivir ${ }^{8}$.

For favipiravir to have its potent antiviral activity, it must be converted to favipiravir-4-ribofuranosyl-5'-tri- 
phosphate (T-705RTP). This is mediated by the action of hypoxanthine-guanine phosphoribosyl-transferase (HGPRT) and cellular nucleotide kinase ${ }^{14,16}$. HGPRT converts T-705 into T-705-4-ribofuranosyl-5'-monophosphate (T-705RMP) directly through one step phosphoribosylation without the need of the rate-limiting nucleoside kinase enzyme. Due to the preferable structural configuration, favipiravir behaves more as guanine mimetic than adenine mimetic during initial activation by HGPRT ( ref. $^{14}$ ). T-705RMP undergoes two more phosphorylation steps into T-705RTP (ref. ${ }^{16}$ ). Although T-705 and T-705RMP also have inhibitory effects, only T-705RTP shows significant and potent inhibition of replication of the virus ${ }^{14}$.

\section{MECHANISMS OF ACTION AGAINST COVID-19}

SARS-CoV2 is an RNA virus containing a singlestrand of positive sense molecule of ribonucleic acid. The genome encodes for several structural, nonstructural (nsp), and accessory proteins. Of particular interest, it encodes for RNA-dependent RNA polymerase ( $\mathrm{RdRp}$ or nsp12) and, additionally, the cofactors (nsp7 and 8), which together form the RNA polymerase complex. Also, it encodes the non-structural protein nsp14 with exonuclease $\mathrm{N}$-terminal domain (ExoN). RdRp is an enzyme responsible for translating SARS-CoV2 genetic material to a negative-strand RNA, which plays a role as a template for protein transcription or synthesis of new gRNAs. Thus, inhibiting RdRp results in the termination of the viral life cycle. Antiviral antimetabolites take advantage of viral RdRp's low fidelity, which is defined as the viral enzymes' ability to discriminate the nucleotide analogs triphosphates from the natural endogenous triphosphates of naturally occurring nucleotides ${ }^{17}$. Coronaviruses polymerases are particularly vulnerable to antimetabolites as they may position them into the active site. This explains why they are the fastest polymerases but at the expense of their decreased fidelity ${ }^{18}$. Remdesivir and favipiravir are RNA polymerase inhibitors, yet they exert their effects differently. These two drugs are direct-acting antivirals that target a particular process of the viral (SARS-CoV2) life cycle ${ }^{18,19}$.

Remdesivir actions are mediated by its active form, triphosphate. It enters in competition with adenosine triphosphate (ATP), the natural substrate for which remdesivir is an analog. Remdesivir incorporates three times more efficiently into the nascent RNA. The incorporation is a random process. The 3'-hydroxyl group makes it possible for the strand to continues to elongate through the formation of phosphodiester bonds with the following nucleoside triphosphate. Three more nucleotides are added downstream the position of the incorporated analog $(i+3)$. Structural modeling predicts a steric clash between the cyano substitution at $\mathrm{C} 1$ of the incorporated analog's sugar and the ser- 861 residue of the RdRp complex at $i+3$. This explains how the incorporated analog prevents the enzyme complex translocation into the $i+4$ position and arrests the strand elongation at the $\mathrm{i}+3$ position and why remdesivir acts as a delayed chain terminator even if this termination is not absolute. The translocational equilibrium shifts towards the $\mathrm{i}+4$ position at higher ATP concentration thus exposing the nucleotide-binding site for further elongation. The resulting full-length RNA chains contain several incorporated analogs and are susceptible to the ExoN enzyme ${ }^{19,20}$. Coronaviruses exonuclease has a 3'-5' proofreading effect. It is responsible for excising and removing antimetabolites embedded in the viral RNA. The potency of remdesivir is retained because of two main factors. The first one is the fact that the three added nucleotides provide partial protection from ExoN. The other is that the embedded GS-441524-MP causes steric hindrance pushing its ribose moiety toward the ions catalytic site of ExoN and inhibits the enzyme activity. However, exonuclease remains able to identify and remove the analog. Essential in this situation is that the rate of GS-441524-TP being incorporated into RNA strain must exceed the rate of its removal by ExoN ( ref. $^{17}$ ). The newly synthesized negative-sense RNA strands containing the embedded analog provide an additional template-dependent mechanism of action. When uridine triphosphate (UTP) approaches to base-pair with the adenosine ana$\log$, its incorporation into the nascent strand is sterically compromised. The polymerase fails to elongate the chain and the termination is seen at the position of UTP incorporation and/or at UTP +1 . Interestingly, the viral RdRp cannot overcome this obstacle by having a higher natural nucleotides triphosphate pool. This mechanism relies on the analog's ability to partially avoid exonuclease proofreading ${ }^{20}$.

Favipiravir actions are also mediated by its triphosphate, T-705RTP. It acts as a universal purine mimetic, competing with both adenosine and guanosine triphosphates (ATP and GTP). This is evident by the decreased efficacy of favipiravir in the presence of increased ATP and GTP concentrations. The exact mechanism of favipiravir actions is controversial and appears to be speciesdependent ${ }^{21}$. For SARS-CoV2, incorporation of T-705RTP into the structure of nascent RNA strand results in mutagenetic events and/or chain termination. In vitro studies using primer-template have shown that the inhibition mechanism depends on whether T-705RTP is incorporated instead of ATP or GTP. Base-pairing with cytidine triphosphate (GTP replacement) is stalled. However, further chain elongation is fast, promoting the accumulation of full-length products. These RNA strands have an increased rate of mutations when guanine is changed to adenine and cytosine to uracil. The increased number of T-705RTP inserted and the increased rate of mutations may be higher than what viral repair machinery can correct. Considering that the SARS-CoV2 genome has a low cytosine content, further lowering by these mutations overwhelms the virus. Additionally, these mutations are lethal because they result in substantial loss of viral-specific infectivity. Contrarily, insertion of T-705RTP opposite to uridine triphosphate (in replacement of ATP) is rapid but results in slow and inefficient chain elongation. After multiple insertions, the synthesis of new RNA is termi- 
nated. However, abruptly terminated products are seen only after consecutive T-705RTP incorporation, which is of a low frequency. This supports lethal mutagenesis as the primary mechanism of RdRp inhibition ${ }^{18}$. Some data have shown that lower favipiravir concentration results in full-length products while lethal mutagenesis predominates. Higher favipiravir concentrations promote consecutive insertions and chain termination ${ }^{21}$.

\section{CLINICAL TRIALS ON REMDESIVIR AND FAVIPIRAVIR IN COVID-19 PATIENTS}

A search was executed using the ClinicalTrials.gov database. The search for clinical trials investigating remdesivir discovered 108 trials (ref. ${ }^{6}$ ). NIH guidelines were also consulted for the selection of trials having the highest impact (Search of Remdesivir \& Covid19 - List results - ClinicalTrials.gov, NIH, 2021 ( ref. $\left.^{22}\right)$ ). Only 52 clinical trials investigating favipiravir were found (Search: Favipiravir \& Covid19 - List results - ClinicalTrials.gov $\left(\right.$ ref. $\left.\left.{ }^{23}\right)\right)$. However, the information provided by some clinical trials not registered in the ClinicalTrials.gov database is also included in this review.

\section{Determination of efficacy and safety of remdesivir - selected clinical trials}

The possibility of using remdesivir in the therapy of COVID-19 was established at the early stages of the pandemic. Preliminary data demonstrated the efficacy of remdesivir in patients infected by the virus, and subsequently, clinical trials were launched to demonstrate its efficacy and safety. One of the most significant trials is the Adaptive COVID-19 Treatment Trial, or ACTT1, conducted by the National Institute of Allergy and Infectious Diseases (NIAID). ACTT-1 is a randomized, placebo-controlled, double-blinded, phase 3 trial performed in 60 trial sites worldwide from February to April 2020 (ClinicalTrials.gov identifier NCT042807056). In this trial, COVID-19 hospitalized cases aged 18 or above were randomized into two groups and stratified according to the gravity of the infection and the place of admission. The dose of remdesivir for one group ( $n=541$ patients) was $200 \mathrm{mg}$ on the first day and then $100 \mathrm{mg} /$ day for 9 days. The control group ( $n=521$ patients) received an intravenous placebo for 10 days. The primary endpoint evaluated was the time necessary for the patient's recovery. The other parameter followed was the clinical status of a patient on the 15 th day after the beginning of the treatment. For both evaluated outcomes, remdesivir showed to be superior over placebo. The authors suggested that remdesivir precluded the development of the infection to become more serious. Authors think that remdesivir may decrease the requirements for the health care system and thus preserve its resources ${ }^{24}$.

Intending to identify the shortest effective duration of treatment using remdesivir, a randomized, open-label, multi-center, phase 3 clinical trial under the sponsorship of Gilead Sciences (ClinicalTrials.gov identifier:
NCT04292899) was performed. The trial, which was launched in March 2020, evaluated two remdesivir regimens in patients with severe COVID-19. Patients received the drug either for 5 or 10 days ( $n=200$ and 197 patients, respectively). The dose of remdesivir was $200 \mathrm{mg}$ on the first day of the treatment and $100 \mathrm{mg} /$ day for 4 or 9 days. Study results supported the efficacy of remdesivir. However, no significant gap between the outcomes of both regimens was registered. Accordingly, a shorter course of therapy was suggested to minimize hospital stay and overcome supply issues. However, the study results are limited by the potential residual confounding caused by the baseline imbalance of the disease severity when the two cohorts of patients are compared ${ }^{25}$.

Despite remdesivir being a promising agent, the interim WHO Solidarity trial results were discouraging. Solidarity trial is an open-label, randomized trial performed from March to October 2020 in 30 countries (ClinicalTrials.gov identifier: NCT04315948). It evaluated the effect of remdesivir and several other drugs on mortality. However, the study design was adaptive, and medications were added and dropped based on the continuously changing data on their efficacy. A total of 11330 hospitalized COVID-19 patients who are above 18 years old were recruited. Patients were assigned to either receiving the local standard of care (SOC) alone or with one of the drugs mentioned above randomly. Remdesivir group $(n=2750)$ received $200 \mathrm{mg} /$ day on the day of admission followed by $100 \mathrm{mg} /$ day during 9 following days, while its control $(n=2708)$ received the SOC alone. The patients were followed up to the death as a primary outcome. Other evaluated parameters were the need for ventilation and the duration of hospital stay. The trial has concluded that these four drugs display no or minimal effect on in-hospital mortality, the necessitation of ventilation, and the length of hospitalization ${ }^{26}$. Another evidence against the use of remdesivir was the trial sponsored by Gilead Sciences with the ClinicalTrials.gov identifier NCT04292730. It was also an open-label, randomized trial that involved 596 subjects with moderate severity of the infection. The participants were above the age of 12 and were hospitalized in 105 hospitals from March to April 2020. One hundred and ninety-seven subjects were treated by a 5-days course of remdesivir (dosed similarly to previously mentioned trials), 194 received a 10-days course, and the remaining 200 were managed with the SOC alone. By day 11, patients' conditions were assessed and analyzed as the primary endpoint, and the adverse effects were reported as a secondary endpoint. Results showed a significant, but of uncertain medical relevance, improvement of the patient's health status with the 5-days course of remdesivir compared with the SOC alone. The longer therapy course did not differ in its outcomes. However, a significantly higher frequency of adverse effects was registered with the prolonged remdesivir therapy compared to the patients receiving remdesivir according to the 5-days protocol. These adverse effects were mild, including hypokalemia, headache, and nausea. Moreover, serious adverse effects were more likely to occur in pa- 
tients managed with the SOC alone compared to those managed with remdesivir for any duration ${ }^{27}$.

\section{Determination of efficacy and safety of favipiravir - selected clinical trials}

Favipiravir is an oral agent with a well-identified safety profile and in vitro activity against SARS-CoV2 RdRp, which represented a valuable option in the treatment of COVID-19. By February 28, 2021, 10986 patients treated with favipiravir in Japan since March 2020 were registered to an observational study database. The researcher observed that the clinical indicators of these patients got significantly better at the 7 th and $14^{\text {th }}$ days $(72.6 \%$ and $86.5 \%$ in mild COVID-19 cases, $63.4 \%$ and $77.2 \%$ in moderate cases, and $46.6 \%$ and $60.4 \%$ in severe cases, respectively). Only 2,841 of those patients reported adverse effects, which were more common in younger patients ${ }^{28}$. The conducted trials on the use of favipiravir are flawed with their designs, methodology, or very small sample sizes. One clinical trial (randomized, open-label, phase 3) was performed in mild/moderate or asymptomatic patients with COVID-19. The goal of this trial was to examine risks related to the use of favipiravir in this subset. It took place from May to July 2020 and involved 150 patients aged 18 to 75 years old. Among those who completed the trial, 70 patients received $1800 \mathrm{mg}$ x 2/day 1 then $800 \mathrm{mg}$ x 2/ days 2-14 along with the local standards of care (SOC), and 68 patients managed with the SOC alone. No meaningful variation was registered regarding the time of viral clearance, the primary outcome assessed, but this was attributed to the limitations of RT-PCR (reverse transcription-polymerase chain reaction) used for the assessments. Besides, patients who received favipiravir achieved clinical cure $40 \%$ faster than those who received the SOC alone ( 3 vs. 5 days) with only transient mild-moderate adverse effects. The study results raised the possibility of outpatient treatment with favipiravir ${ }^{29}$. Another trial studying a similar subset of COVID-19 patients but aged 16 and above compared the effect of early administration of favipiravir at the day of participation $(n=36)$ to its effects if administered at day 6 of participation $(n=33)$. Notwithstanding the absence of any significant clinical gap between the two groups in such parameters as viral clearance, it has been suggested that earlier intervention with the drug at earlier stages of the disease may be more beneficial. However, the trial results are limited by the very small number of participants who continued the trial ${ }^{30}$. Additionally, an open-label, nonrandomized trial was started on January 30, 2020 (ref. $^{31}$ ). At that date, 35 patients were assigned to receive $1600 \mathrm{mg} \times 2$ /day 1 and then $600 \mathrm{mg} \times 2$ /day 2-14 (for 13 days). The control was a selected group of patients who were treated with the combination of 400 $\mathrm{mg}$ of lopinavir and $100 \mathrm{mg}$ of ritonavir (LPV/RTV) twice per day $/ 7$ days preceding the therapy with favipiravir $(n=$ 45 patients). Additionally, interferon- $\alpha 1 \mathrm{~b}$ was administered to patients in both groups (60 mcg twice per day). Treatment regimens continued for 14 days or until viral clearance. It has been found that favipiravir has a greater effect on shortening the time until viral clearance than LPV/RTV ( 4 vs. 11 days). Furthermore, a better therapeutic outcome was shown by chest computed tomography scans as the faster and greater improvement was observed at day 14 in patients treated with favipiravir, an effect in which the accelerated viral clearance has contributed ${ }^{31}$.

\section{Side effects and contraindications of remdesivir and favipiravir}

Several adverse reactions have been reported in trials in which COVID-19 patients received remdesivir or favipiravir. Both drugs induced liver enzymes, including alanine and aspartate aminotransferases. Remdesivir also induced bilirubin and resulted in more drug discontinuation due to liver injury when compared to placebo. However, several cases of liver injury were reported in COVID-19 patients making it harder to discriminate whether the elevation in liver enzymes is related to the disease or the use of remdesivir or favipiravir ${ }^{32-34}$. Moreover, a higher percentage of patients on remdesivir discontinued the drug in a randomized clinical trial due to gastrointestinal side effects, including nausea, vomiting, and anorexia, or respiratory adverse events, such as acute respiratory distress syndrome and respiratory failure. Cardiovascular and renal toxicities were also reported in patients treated with remdesivir for COVID-19. These include hypotension, atrial fibrillation, acute renal failure, hematuria, and hypernatremia. Other adverse events include rash, pyrexia, and multi-organ damage. These toxicities cannot be attributed solely to the use of remdesivir since COVID-19 by itself is involved in multi-organ damage ${ }^{32}$. Hypersensitivity and infusion-related reactions were reported during and after the intravenous administration of remdesivir. On the other hand, only a few other minor adverse effects were reported in favipiravir-treated patients with COVID-19. The most common of which is a dose-dependent elevation in serum uric acid. However, hyperuricemia was not associated with any clinical events, i.e., gout attacks. Other side effects include diarrhea and decreased neutrophil count. Psychiatric symptoms and QT-prolongation association with favipiravir is uncertain ${ }^{34}$. Remdesivir and favipiravir labeling recommend against their use in severe renal impairment $(\mathrm{eCrCl}<30 \mathrm{~mL} / \mathrm{min}$ - estimated creatinine clearance). Additionally, favipiravir is also contraindicated in severe liver impairment. In terms of reproductive toxicities, favipiravir carries potential teratogenicity and embryotoxicity, as observed in animal studies. This led the Japanese drug safety bureau approval to recommend a warning against the use of favipiravir in females with child-bearing potential. Moreover, favipiravir is distributed into sperms, extending the caution to males with partners in their reproductive age. Effective contraceptives are strongly recommended during treatment with favipiravir and for 7 days after treatment, while pregnant women are prohibited from taking this drug. Remdesivir is advantageous in this context as it appears to be safe during pregnancy. This is evident by previous studies on the use of remdesivir against the Ebola virus ${ }^{33,34}$. 


\section{CURRENT TREATMENTS OF COVID-19 PATIENTS USING REMDESIVIR OR FAVIPIRAVIR: WHICH ONE IS OF GREATER BENEFIT?}

To date, remdesivir remains the only antiviral agent approved by the FDA for the therapy of COVID-19 $\left(\right.$ ref. $\left.^{35}\right)$. It was approved under the brand name Veklury on October 22, 2020, to be used in "hospitalized COVID-19 adults and pediatrics patients above 12 years of age and weighing at least $40 \mathrm{~kg}$ ". The FDA-recommended regimen for such patients is $200 \mathrm{mg}$ of remdesivir intravenously (loading dose) on day 1 and then $100 \mathrm{mg}$ /day at the days 2-5 or 2-10. The FDA also kept the validity of remdesivir emergency use approval (from May 1, 2020) for children patients treated in a hospital with the weight of 3.5 to 40 $\mathrm{kg}\left(\right.$ ref. ${ }^{35}$ ). The guidelines approved by IDSA (Infectious Disease Society of America) currently prefer remdesivir over no antiviral therapy in severe COVID-19 persons. The course of remdesivir ( 5 days) is recommended for hospitalized patients requiring oxygen therapy. However, IDSA guidelines recommend against its use in severe cases requiring the use of mechanical ventilation. The same recommendation was issued regarding COVID-19 patients on extracorporeal membrane oxygenation (ECMO), and even for other patients with severe COVID-19 (that may not require oxygen supplementation) ( ref. $^{36}$ ). Additionally, according to National Institute of Health guidelines, it is reasonable to give remdesivir for moderate cases at high risk of disease progression and in pregnant patients if clinically indicated ${ }^{6}$. On the other hand, favipiravir is not approved by the U.S. FDA; neither has any recommendations for or against its use in IDSA or NIH (ref. ${ }^{6,36}$ ).

The use of remdesivir was also authorized by the regulatory institutions of Japan for the treatment of COVID-19 on May 7, 2020. The Japanese latest treatment guideline recommends administering remdesivir at the same FDA-recommended doses for severe cases with oxygen saturation $\leq 94 \%$ or requiring oxygen supplementation. Additionally, these agencies suggested the off-label use of favipiravir in the setting of clinical studies ${ }^{37}$. Patients who received favipiravir in Japan were registered in an observational study database, as previously mentioned ${ }^{28}$.

Remdesivir and favipiravir are included in several local protocols worldwide ${ }^{38}$. For example, the Thailand clinical practice guideline recommends favipiravir for the therapy of COVID-19 as the primary antiviral agent. It is used alone, both in adults and children with the prognosis of the highly risky disease progression or with corticosteroids in cases of hypoxia or progressed pulmonary infiltrates. Favipiravir regimen suggested for adults is 1800 mg x 2 per one day and then $800 \mathrm{mg}$ x 2/days 2-5 or 2-10. The guidelines suggest remdesivir as an alternative when favipiravir cannot be used, such as in pregnancy and if oral administration of drugs is not possible (Covid-19 Response Committee, 2021) (ref. ${ }^{38}$ ). Another example is the COVID-19 Treatment Protocol approved by the Ministry of Health of Saudi Arabia ${ }^{39}$, which recommends the application of both drugs although they are not yet registered by the Saudi Food and Drug Administration.
At doses reflecting the protocols mentioned above, favipiravir is recommended in mild to moderate cases, favipiravir or remdesivir in severe cases, and remdesivir with or without corticosteroids in critical cases such as in adults suffering from acute respiratory distress syndrome. The therapy should last from 7 to 10 days with favipiravir. The treatment with remdesivir should last from 5 to 10 days ${ }^{39}$.

In Kuwait, remdesivir is the only antiviral agent used in fighting COVID-19 disease. According to Kuwait COVID-19 Adult Task Force Management Recommendations, remdesivir is indicated for patients with COVID-19 pneumonia on oxygen but not for patients requiring high-flow oxygen, ECMO for 5 days, or even non-invasive or mechanical ventilation. However, in patients on ECMO or mechanical ventilation, remdesivir treatment can last up to 10 days ${ }^{40}$. By contrast, favipiravir was only used in the context of a clinical trial AVIGAN (ClinicalTrials.gov; identifier: NCT04529499) that started August 20, 2020 (Clinical trial evaluating the efficacy and safety of favipiravir in moderate to severe COVID-19 patients). Dr. Reddy's Laboratories Ltd, the sponsor, announced the trial's termination on January 27, 2021, and stated that the data obtained from Kuwait did not demonstrate any clear improvement in patients' hypoxia ${ }^{41}$.

Taking the results of trials investigating the efficacy and safety of remdesivir and favipiravir altogether, it appears that in comparison to favipiravir, remdesivir is more beneficial for patients with COVID-19. Subjects with the severe disease on remdesivir demonstrated a better clinical course of the disease without increased serious adverse events when compared to those not receiving the drug. Moreover, remdesivir administration resulted in a shorter median time to recovery and decreased need for mechanical ventilation in this population. However, these effects were not observed in patients with mild-moderate cases or severe cases on mechanical ventilation or ECMO $\left(\right.$ ref. $\left.{ }^{36}\right)$. In contrast, the evidence in favor of favipiravir is less significant. It indicates the possibility of outpatient treatment and covers a different spectrum of patients with mild-moderate cases of the disease. Management with favipiravir was associated with faster improvement in chest radiography and faster clinical cure ${ }^{29,31}$. Favipiravir treatment resulted in decreased frequency of adverse effects when compared to remdesivir, but the high pill burden (18 tablets in two divided doses on day 1 and 4 tablets twice a day for the remaining duration of treatment) is disadvantageous ${ }^{32,34}$. More clinical investigations are necessary for verification, confirmation, and support of the benefits that favipiravir brings to the patients during this pandemic.

\section{CONCLUSION}

COVID-19 pandemic is a major threat to people, and continuous efforts are required to fight the disease and abate its consequences. During the search for a safe and efficacious therapy of the disease, remdesivir and favipiravir were investigated. They are two antiviral an- 
timetabolites that work by inhibiting RNA polymerase, thus interrupting viral replication. Chemical structures of remdesivir and favipiravir enable them to mimic adenine (remdesivir) and both purines (favipiravir). Both agents are prodrugs that are activated intracellularly and incorporated in the newly synthesized RNA instead of the natural nucleotides. After incorporation into the RNA strand, they exert their effects through numerous mechanisms. Current data obtained from clinical trials on remdesivir are more significant and they are reflected in the current global guidelines that recommend its administration in specific severe cases of the disease. Remdesivir also currently serves as the only antiviral drug approved by FDA (USA) for use in COVID-19 patients. Its clinical effects include decreasing the time to recovery and minimizing the need for ventilation. In terms of safety, remdesivir is associated with an elevation in liver enzymes, deterioration of renal function, hypersensitivity reactions, and several other toxicities. Although favipiravir showed some advantages (especially in mild-moderate cases of the disease) and is relatively safe compared to remdesivir, the evidence about its use is uncertain. Benefits of favipiravir include accelerating clinical cure and chest improvement assessed by radiography. Drawbacks of favipiravir include its potential teratogenicity and high pill burden. Larger, well-designed clinical trials are needed to fully address favipiravir potentials in the treatment of COVID-19, especially in outpatient settings. However, both drugs bring benefits to COVID-19 patients. However, remdesivir is currently of more significant utility compared to favipiravir.

\section{Search strategy and selection criteria}

The review is based on selective literature searches in PubMed, with remdesivir and/or favipiravir being always the word that any search was based on. Other public domain documents and web pages (i.e., from the WHO, NIH, CNN, ClinicalTrials database pages etc.) were also used.

Acknowledgment: The authors are grateful to Kuwait University for the support.

Authors' contribution: FMAA: contributed mainly to the literature search and original write-ups; LN: originated the idea for this manuscript, participated in writing the manuscript and final revisions; AA: contributed to developing the manuscript idea, final readings, and revisions; AFAT: help to develop the original idea, participated in writing parts of the manuscript, final readings, and revisions. Conflict of interest: The authors declare no conflict of interest.

\section{REFERENCES}

1. World Health Organization. Timeline: WHO's COVID-19 response 2020 [accessed 2021 September 12]. Available from: https://www. who.int/emergencies/diseases/novel-coronavirus-2019/interactivetimeline.

2. Dhama K, Khan S, Tiwari R, Sircar S, Bhat S, Malik YS, Singh KP, Chaicumpa W, Bonilla-Aldana DK, Rodriguez-Morales AJ. Coronavirus
Disease 2019-COVID-19. Clin Microbiol Rev 2020;33:e00028-20. doi: $10.1128 / \mathrm{cmr} .00028-20$

3. Kadam S B, Sukhramani GS, Bishnoi P, Pable AA, Barvkar VT. SARSCoV-2, the pandemic coronavirus: Molecular and structural insights. J Basic Microbiol 2021;61:180-202. doi: 10.1002/jobm.202000537

4. Ni W, Yang X, Yang D, Bao J, Li R, Xiao Y, Hou C, Wang H, Liu J, Yang D, Xu Y, Cao Z, Gao Z. Role of angiotensin-converting enzyme 2 (ACE2) in COVID-19. Crit Care 2020;24:422. doi: 10.1186/s13054-020-03120-0

5. Delikhoon M, Guzman MI, Nabizadeh R, Norouzian Baghani A. Modes of transmission of severe acute respiratory syndrome-coronavirus-2 (SARS-CoV-2) and factors influencing on the airborne transmission: A review. Int J Environ Res Public Health 2021;18:395. doi: 10.3390/ ijerph18020395

6. National Institutes of Health. COVID-19 Treatment Guidelines. 2021 [accessed 2021 September 12]. Available from: https://www.covid19treatmentguidelines.nih.gov/

7. Jordheim LP, Durantel D, Zoulim F, Dumontet C. Advances in the development of nucleoside and nucleotide analogues for cancer and viral diseases. Nature Rev Drug Discovery 2013;12:447-464. doi: $10.1038 / \mathrm{nrd} 4010$

8. Eastman RT, Roth JS, Brimacombe KR, Simeonov A, Shen M, Patnaik $S$, Hall MD. Remdesivir: A review of its discovery and development leading to emergency use authorization for treatment of COVID-19. ACS Central Science 2020;6:672-83. doi: 10.1021/acscentsci.0c00489

9. Yates MK, Seley-Radtke KL. The evolution of nucleoside analogue antivirals: A review for chemists and non-chemists. Part 1: Early structural modifications to the nucleoside scaffold. Antiviral Res 2018;154:66-86. doi: 10.1016/j.antiviral.2018.04.004

10. Jordan PC, Stevens SK, Deval J. Nucleosides for the treatment of respiratory RNA virus infections. Antiviral Chem Chemotherapy 2018;26:204020661876448. doi: 10.1177/2040206618764483

11. Vardanyan R, Hruby V. Chapter 34 - Antiviral Drugs. In Vardanyan R, Hruby V., editors. Synthesis of Best-Seller Drugs, Elsevier (Academic Press imprint) 2016, pp. 687-736. doi: 10.1016/b978-0-12-411492$0.00034-1$

12. Yates MK, Seley-Radtke KL. The evolution of antiviral nucleoside analogues: A review for chemists and non-chemists. Part II: Complex modifications to the nucleoside scaffold. Antiviral Res 2019:162:5-21. doi: 10.1016/j.antiviral.2018.11.016

13. Umar $Y$. Theoretical studies of the rotational and tautomeric states, electronic and spectroscopic properties of favipiravir and its structural analogues: a potential drug for the treatment of COVID-19. J Taibah University for Science 2020;14:1613-25. doi: 10.1080/16583655.2020.1848982

14. Naesens L, Guddat LW, Keough DT, Van Kuilenburg A B, Meijer J, Vande Voorde J, Balzarini J. Role of human hypoxanthine-guanine phosphoribosyltransferase in activation of the antiviral agent T-705 (favipiravir). Mol Pharmacol 2013;84:615-29. doi: 10.1124/ mol.113.087247

15. Liu P, Sharon A, Chu CK. Fluorinated nucleosides: Synthesis and biological implication. J Fluorine Chem 2008;129:743-66. doi: 10.1016/j. jfluchem.2008.06.007

16. Furuta $Y$, Takahashi K, Kuno-Maekawa M, Sangawa H, Uehara S, Kozaki K, Nomura N, Egawa H, Shiraki K. Mechanism of action of T-705 against Influenza Virus. Antimicrobial Agents Chemother 2005;49:981-86. doi: 0.1128/aac.49.3.981-986.2005

17. Shannon A, Le NT-T, Selisko B, Eydoux C, Alvarez K, Guillemot J-C, Decroly E, Peersen O, Ferron F, Canard B. Remdesivir and SARSCoV-2: Structural requirements at both nsp12 RdRp and nsp14 exonuclease active-sites. Antiviral Res 2020;178:104793. doi: 10.1016/j. antiviral.2020.104793

18. Shannon A, Selisko B, Le N-T-T, Huchting J, Touret F, Piorkowski G, Fattorini V, Ferron F, Decroly E, Meier C, Coutard B, Peersen O, Canard B. Rapid incorporation of Favipiravir by the fast and permissive viral RNA polymerase complex results in SARS-CoV-2 lethal mutagenesis. Nat Commun 2020;11:4682. doi: 10.1038/s41467-020-18463-z

19. Gordon CJ, Tchesnokov EP, Woolner E, Perry JK, Feng JY, Porter DP, Götte M. Remdesivir is a direct-acting antiviral that inhibits RNAdependent RNA polymerase from severe acute respiratory syndrome coronavirus 2 with high potency. J Biol Chem 2020;295:6785-97. doi: 10.1074 /jbc.ra120.013679

20. Tchesnokov EP, Gordon C J, Woolner E, Kocinkova D, Perry JK, Feng JY, Porter DP, Götte M. Template-dependent inhibition of coronavirus RNA-dependent RNA polymerase by remdesivir reveals a second 
mechanism of action. J Biol Chem 2020;295:16156-65. doi: 10.1074/ jbc.ac120.015720

21. Delang L, Abdelnabi R, Neyts J. Favipiravir as a potential countermeasure against neglected and emerging RNA viruses. Antiviral Res 2018;153:85-94. doi: 10.1016/j.antiviral.2018.03.003

22. Search of: Remdesivir | Covid19 - List results - ClinicalTrials.gov. ClinicalTrials.gov. [accessed 2021 June 10]. Available from: https:// clinicaltrials.gov/ct2/results?cond=Covid19\&term=remdesivir\&cnt $r y=\&$ state $=\&$ city $=\&$ dist $=\&$ Search $=$ Search

23. The search of Favipiravir | Covid19- List results - ClinicalTrials.gov. ClinicalTrials.gov. https://clinicaltrials.gov/ct2/results?cond=Covid 19\&term=favipiravir\&cntry=\&state $=\&$ city $=\&$ dist $=\&$ Search $=$ Search

24. Beigel JH, Tomashek KM, Dodd LE, Mehta AK, Zingman BS, Kalil AC, Hohmann E, Chu HY, Luetkemeyer A, Kline S, Lopez de Castilla D, Finberg RW, Dierberg K, Tapson V, Hsieh L, Patterson TF, Paredes R, Sweeney DA, Short WR, Touloumi G, Lye DC, Ohmagari N, Oh M, Ruiz-Palacios GM, Benfield T, Fätkenheuer G, Kortepeter MG, Atmar RL, Creech CB, Lundgren J, Babiker AG, Pett S, Neaton JD, Burgess TH, Bonnett T, Green M, Makowski M, Osinusi A, Nayak S, Lane HC. Remdesivir for the Treatment of Covid-19 - Final Report. N Engl J Med 2020;383:1813-26. doi: 10.1056/nejmoa2007764

25. Goldman JD, Lye DCB, Hui DS, Marks KM, Bruno R, Montejano R, Spinner CD, Galli M, Ahn M-Y, Nahass RG, Chen Y-S, SenGupta D, Hyland RH, Osinusi AO, Cao H, Blair C, Wei X, Gaggar A, Brainard DM, Towner WJ, Muñoz j, Mullane KM, Marty FM, Tashima KT, Diaz $\mathrm{G}$, Subramanian A. Remdesivir for 5 or 10 days in patients with severe Covid-19. N Engl J Med 2020;383:1827-37. doi: 10.1056/ NEJMoa2015301

26. Pan $\mathrm{H}$, Peto R, Henao-Restrepo A-M, Preziosi M-P, Sathiyamoorthy V, Abdool Karim Q, Alejandria MM, Hernández García C, Kieny M-P, Malekzadeh R, Murthy S, Reddy KS, Roses Periago M, Abi Hanna P, Ader F, Al-Bader AM, Alhasawi A, Allum E, Alotaibi A, Alvarez-Moreno CA, Appadoo S, Asiri A, Aukrust P, Barratt-Due A, Bellani S, Branca M, Cappel-Porter HBC, Cerrato N, Chow TS, Como N, Eustace J, García PJ, Godbole S, Gotuzzo E, Griskevicius L, Hamra R, Hassan M, Hassany $M$, Hutton D, Irmansyah I, Jancoriene L, Kirwan J, Kumar S, Lennon P, Lopardo G, Lydon P, Magrini N, Maguire T, Manevska S, Manuel O, McGinty S, Medina MT, Mesa Rubio ML, Miranda-Montoya MC, Nel J, Nunes EP, Perola M, Portolés A, Rasmin MR, Raza A, Rees $H$, Reges PPS, Rogers CA, Salami K, Salvadori MI, Sinani N, Sterne JAC, Stevanovikj M, Tacconelli E, Tikkinen KAO, Trelle S, Zaid H, Røttingen J-A, Swaminathan S. Repurposed antiviral drugs for Covid-19Interim WHO Solidarity Trial results. N Engl J Med 2021;384:497-511. doi: 10.1056/NEJMoa2023184

27. Spinner CD, Gottlieb RL, Criner GJ, Arribas López JR, Cattelan AM, Soriano Viladomiu A, Ogbuagu O, Malhotra P, Mullane KM, Castagna A, Chai LYA, Roestenberg M, Tsang OTY, Bernasconi E, Le Turnier P, Chang SC, SenGupta D, Hyland RH, Osinusi AO, Cao H, Blair C, Wang H, Gaggar A, Brainard DM, McPhail MJ, Bhagani S, Ahn MY, Sanyal AJ, Huhn G, Marty FM. Effect of remdesivir vs standard care on clinical status at 11 days in patients with moderate COVID-19. JAMA 2020;324:1048-57. doi: 10.1001/jama.2020.16349

28. Doi $Y$, Kondo $M$, Ando $M$, Kuwatsuka $Y$, Ishihara T. Favipiravir Observational Study Interim Report 3. Fujita Health University, 2021, [accessed 2021 September 12]. Available from: https://www.kansensho.or.jp/uploads/files/topics/2019ncov/covid19_favip_2100419_ eng.pdf

29. Udwadia ZF, Singh P, Barkate H, Patil S, Rangwala S, Pendse A, Kadam J, Wu W, Caracta CF, Tandon M. Efficacy and safety of favipiravir, an oral RNA-dependent RNA polymerase inhibitor, in mild-to-moderate COVID-19: A randomized, comparative, open-label, multicenter, phase 3 clinical trial. Int J Infect Dis 2021;103:62-71. doi: 10.1016/j. ijid.2020.11.142

30. Doi Y, Hibino M, Hase R, Yamamoto M, Kasamatsu Y, Hirose M, Mutoh Y, Homma Y, Terada M, Ogawa T, Kashizaki F, Yokoyama T, Koba H, Kasahara H, Yokota K, Kato H, Yoshida J, Kita T, Kato Y, Kamio T, Kodama N, Uchida Y, Ikeda N, Shinoda M, Nakagawa A, Nakatsumi H, Horiguchi T, Iwata M, Matsuyama A, Banno S, Koseki T, Teramachi M, Miyata M, Tajima S, Maeki T, Nakayama E, Taniguchi S, Lim CK, Saijo M, Imai T, Yoshida H, Kabata D, Shintani A, Yuzawa Y, Kondo M. A pro- spective, randomized, open-label trial of early versus late Favipiravir therapy in hospitalized patients with COVID-19. Antimicrob Agents Chemother 2020;64:e01897-20. doi: 10.1128/AAC.01897-20

31. Cai Q, Yang M, Liu D, Chen J, Shu D, Xia J, Liao X, Gu Y, Cai Q, Yang $Y$, Shen C, Li X, Peng I, Huang D, Zhang J, Zhang S, Wang F, Liu J, Chen L, Chen S, Wang Z, Zhang Z, Cao R, Zhong W, Liu Y, Liu L. Experimental treatment with Favipiravir for COVID-19: An open-label control study. Engineering (Beijing) 2020;6:1192-198. doi: 10.1016/j. eng.2020.03.007

32. Fan $\mathrm{Q}$, Zhang B, Ma J, Zhang S. Safety profile of the antiviral drug remdesivir: An update. Biomed \& Pharmacotherapy 2020;130:110532. doi: 10.1016/j.biopha.2020.110532

33. Joshi S, Parkar J, Ansari A, Vora A, Talwar D, Tiwaskar M, Patil S, Barkate H. Role of favipiravir in the treatment of COVID-19. Int J Infect Dis 2021;102:501-8. doi: 10.1016/j.ijid.2020.10.069

34. Agrawal U, Raju R, Udwadia ZF. Favipiravir: A new and emerging antiviral option in COVID-19. Med J Armed Forces India 2020;76(4):37076. doi: 10.1016/j.mjafi.2020.08.004

35. US Food and Drug Administration. FDA's approval of Veklury (remdesivir) for the treatment of COVID-19, (2020, October 22), [accessed 2021 September 12]. Available from: https://www.fda.gov/drugs/ drug-safety-and-availability/fdas-approval-veklury-remdesivirtreatment-covid-19-science-safety-and-effectiveness

36. Bhimraj A, Morgan RL, Shumaker AH, Lavergne V, Baden L, Cheng VC, Edwards KM, Gandhi R, Gallagher J, Muller WJ, O'Horo JC, Shoham S, Murad MH, Mustafa RA, Sultan S, Falck-Ytter Y. Infectious Diseases Society of America Guidelines on the Treatment and Management of Patients with COVID-19. Infectious Diseases Society of America, 2021. [accessed 2021 September 13]. Available from: https://www. idsociety.org/practice-guideline/covid-19-guideline-treatment-andmanagement/

37 Takuya A, Mugen U, Norio O, Yasuyuki K, Akihiko K, Satoshi K, Toru K, Masayuki S, Koichi T, Shu H, Ryota H, Jiro F, Hiroshi F, Hiroshi M, Masaya Y, Akihito U. (2020). Clinical management of patients with COVID-19: A guide for front-line healthcare workers (Version 2.1). In Ministry of Health, Labour and Welfare of Japan. https://www.mhlw. go.jp/content/000646531.pdf

38. Covid-19 Response Committee. Guidelines on clinical practice, diagnosis, treatment, and prevention of healthcare-associated infection for COVID-19. Department of Medical Services, Ministry of Public Health, Thailand, 2021 May 6. https://ddc.moph.go.th/viralpneumonia/eng/file/guidelines/g_CPG_06may21.pdf

39. Ministry of Health, Saudi Arabia, 2021 May 19. Saudi MoH Protocol for Patients Suspected of/Confirmed with COVID-19: Supportive care and antiviral treatment of suspected or confirmed COVID-19 infection (Version 2.9). [accessed 2021 September 12]. Available from: https://www.moh.gov.sa/en/Ministry/MediaCenter/Publications/ Pages/covid19.aspx\#

40. Al-Ahmad M, Al-Rasheed M, Alhelal B, Alghareeb $\mathrm{H}$, Alsayegh A Alhassawi A, Al-Fares AA, Almajed A, Alhajeri $\mathrm{H}$, Alazemi S, Bulbanat B, Al-Mazeedi N, Alroughani R, Alzafiri A, Al-Ozairi E, Alenezi M, Alkandari A, Abdullah AA, Altourah L. Management of COVID-19 in Adults: Kuwait Adult Task Force Recommendation (1st ed.). Kuwait Ministry of Health. A clinical trial evaluating the efficacy and safety of Favipiravir in moderate to severe COVID-19 patients. (n.d.). ClinicalTrials.gov. Retrieved June 28, 2021, from https://clinicaltrials.gov/ct2/show/NCT04529499

41. Dr. Reddy's Laboratories Ltd., 27 January 2021. Dr. Reddy's and GRA announce Avigan Pivotal Studies Update Study for hospitalized moderate to severe cases in Kuwait terminated while study for out-patient mild to moderate cases continues in North America. https://www.drreddys.com/media/928938/2021-01-avigan-trialupdate_v1.pdf

42. Remdesivir. Pubchem.ncbi.nlm.nih.gov, 2021, [accessed 2021 September 12]. Available from: https://pubchem.ncbi.nlm.nih.gov/ compound/Remdesivir.

43. Favipiravir. Pubchem.ncbi.nlm.nih.gov, 2021, [accessed 2021 September 12]. Available from: https://pubchem.ncbi.nlm.nih.gov/ compound/492405 